\title{
Adolescent Idiopathic Torticollis: Morphological Vertebral Chang- es of the Spinal Canal and the Spinal Cord Position: A Case Report
}

\author{
Pedro Gutiérrez Carbonell* and Ruiz Miñana E \\ Department of Orthopedic Surgery and Traumatology, General University Alicante Hospital, Spain
}

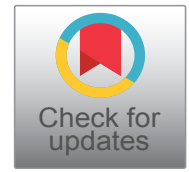

*Corresponding author: Pedro Gutiérrez Carbonell, Department of Orthopedic Surgery and Traumatology, General University Alicante Hospital, Paraje Ledua E-25, 03660-Novelda, Spain, Tel: 00-34-606-468-139, Fax: 00-34-965-242-454,

E-mail: gutierrez_ped@gva.es

\begin{abstract}
Cervical spinal canal deformities can be caused by muscular deforming forces, as in the case of a 10-year-old male with inveterate left torticollis. Atlas and axis bone deformities were observed, including subluxation of the odontoid apophysis, hypertrophy of the anterior arch of the atlas, hypoplasia of the posterior arches of atlas, and deformation of the spinal canal accompanied by an eccentric position of the spinal cord. After tenotomy of Sternocleidomastoid Muscle (SCM) and 5-years of follow-up, the deformities were unchanged. Idiopathic scoliosis, is a 3D deformity, and has similar pattern spinal deformities: Thinning, asymmetry of the pedicles and vertebral laminae and spinal cord displacement toward the concavity of the scoliosis. Persistent muscular deforming forces on the neurocentral cartilage can be sources of structural deformity in vertebral and cervical deformities.
\end{abstract}

\section{Keywords}

Idiopathic congenital torticollis, CT vertebral study, MR-scanning cervical, Morphological vertebral changes, Anomalous spinal cord position

\section{Introduction}

Torticollis is a sign and symptom, not a diagnosis; it requires the formulation of a wide differential diagnosis. In this differential diagnosis are consulted: neurosurgeon, pediatric neurologist, oculist and radiologist. In this case report we have applied algorithm, published in literature [1]. The most common type of torticollis is childhood congenital muscular torticollis. Less common are those types associated with congenital anomalies of the cervical spine (Klippel-Feil Syndrome), gastroesophageal reflux disease
(Sandifer Syndrome), traumatic or oropharyngeal inflammation (Grisel's Syndrome), anomalies of the brain stem and cervical spine (syringomyelia, diastematomyelia and Arnold-Chiari malformation) or tumors of the posterior cranial fossa (astrocytoma and ependymoma) [1-5]. After this wide differential diagnosis was made, orthopedic and occasionally surgical treatment of torticollis with muscular etiology is performed, preferably, within the first year of life, because after this age facial deformities are irreversible. It is unusual for torticollis not to be resolved by the teenage years, but this patient, are an immigrant person without previous treatment. We report a clinical case of idiopathic torticollis with vertebral bone deformities of the cervical spinal canal and deformities in the spinal cord position relative to the canal, which were irreversible despite tenotomy of the Sternocleidomastoid Muscle (SCM). The deformities observed in this patient with torticollis have not been described previously in the literature.

\section{Case Report}

This patient study was approved by the Ethics Committee and was conducted following completion of the corresponding informed consent. A 10-year-old male

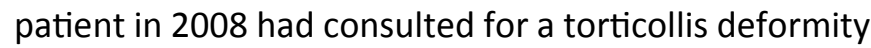
that had originated during early childhood. This delay in first diagnosis is because patient is inmigrant and without diagnosis or previous treatment. Patient had classical deformities of torticollis: Shortening of SCM muscle, lateral inclination of the neck, smaller and higher eye, contralateral torsion with chin rising. Moreover, the patient also presented with a left-side facial defor- 


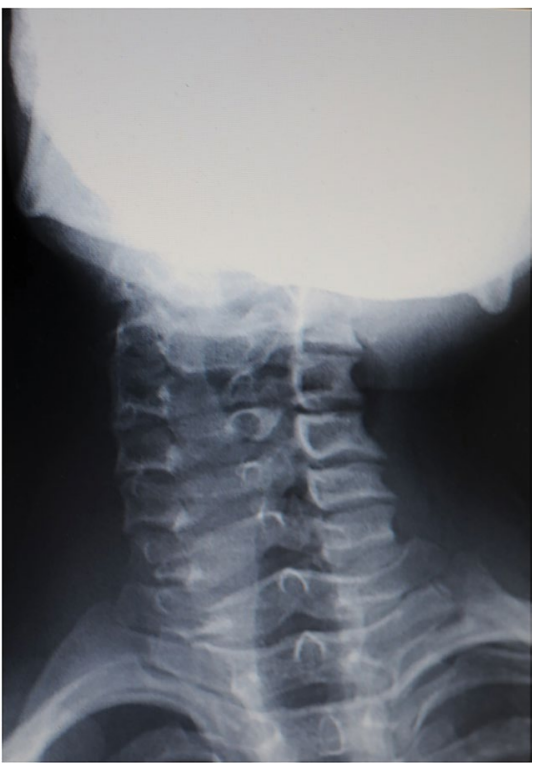

A

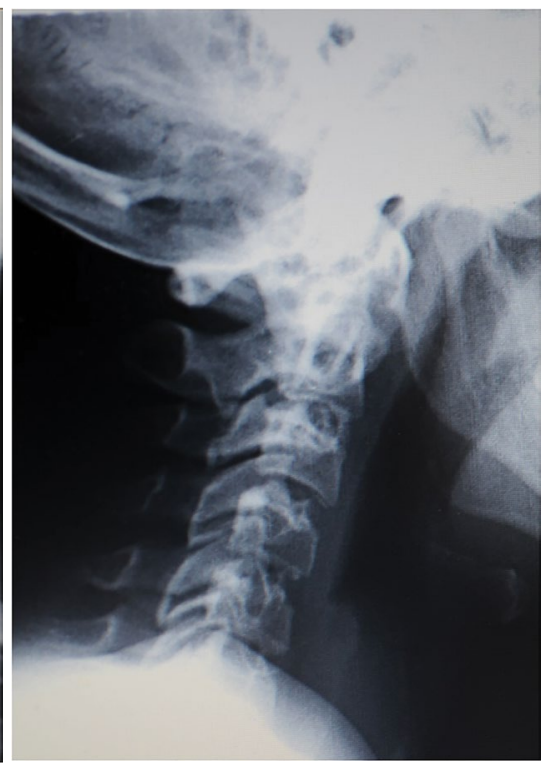

B

Figure 1: Radiographs of the a) Anteroposterior; b) Lateral cervical spine. No anomalies of segmentation or rachischisis of the vertebral arches were observed.
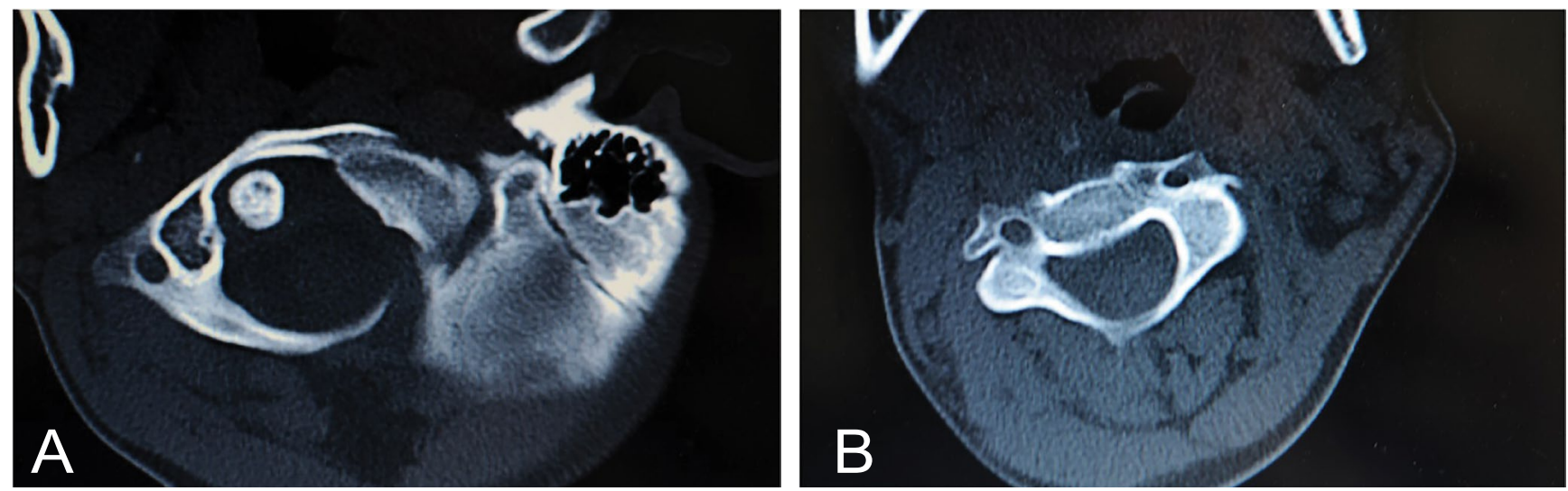

Figure 2: CT: a) Odontoid apophysis asymmetry and right anterior vertebral hemi-arch hypoplasia; b) Spinal canal asymmetry of vertebral posterior arches: Flattening on the right side and convexity on the left.

mity. The patient was not referred for any traumatic or infectious etiology of the oropharynx or for digestive or renal diseases. It was made a wide differential diagnosis, especially for to exclude nonmuscular causes of torticollis, presents in $18 \%$ of patients [1], Klippel-Feil anomalies (30\%), posterior fossa tumors, syringomyelia or Arnold-Chiari malformation (51\%) [1,2]. Additionally, neurologic exploration was done by a pediatric neurologist. Problems like dystonia, ataxia or abnormal deep tendon reflexes in upper and lower limbs did not exist. Also, osteocongenital neurological pathologies of the spinal cord and brain tumor were excluded by a pediatric neurologist. After these exclusions were made, we confirmed a diagnosis of inveterate congenital muscular torticollis. Preoperative studies, radiographic imaging, Computed Axial Tomography (CT) scans and Magnetic Resonance Imaging (MRI) of the cervical spine and skull (Figure 1a, Figure 1b, Figure 2a, Figure 2b, Figure 3a, Figure 3b, Figure 4a, Figure 4b) highlighted Klippel-Feil
Syndrome, fusion defects of the vertebral arches of the atlas, diastematomyelia and hypoplasia of the occipital condyles. CT images demonstrated, deformity of the anterior arch of the atlas was observed, consisting of hypoplasia of the right half and deformity and hypoplasia of the posterior arches $\mathrm{C} 1$ and $\mathrm{C} 2$ with concavity of the right posterior hemi-arch and convexity of the left arch, causing asymmetry of the spinal canal. AdditionalIy, subluxation of the odontoid apophysis toward a right lateral mass of the atlas was observed (Figure 2a, Figure 3a). MRI showed deformity with extreme narrowing of the odontoid apophysis and irregular formation of the spinal canal with displacement of the spinal cord in the direction of the concavity of the torticollis (Figure $4 a$, Figure 4b). In 2009, unipolar distal tenotomy of the left SCM was performed, followed by orthotics and rehabilitation with notable improvement in cervical mobility but with minimal effect on facial asymmetry. In the 5 -year postoperative $\mathrm{CT}$ and $\mathrm{MRI}$, there were no signif- 

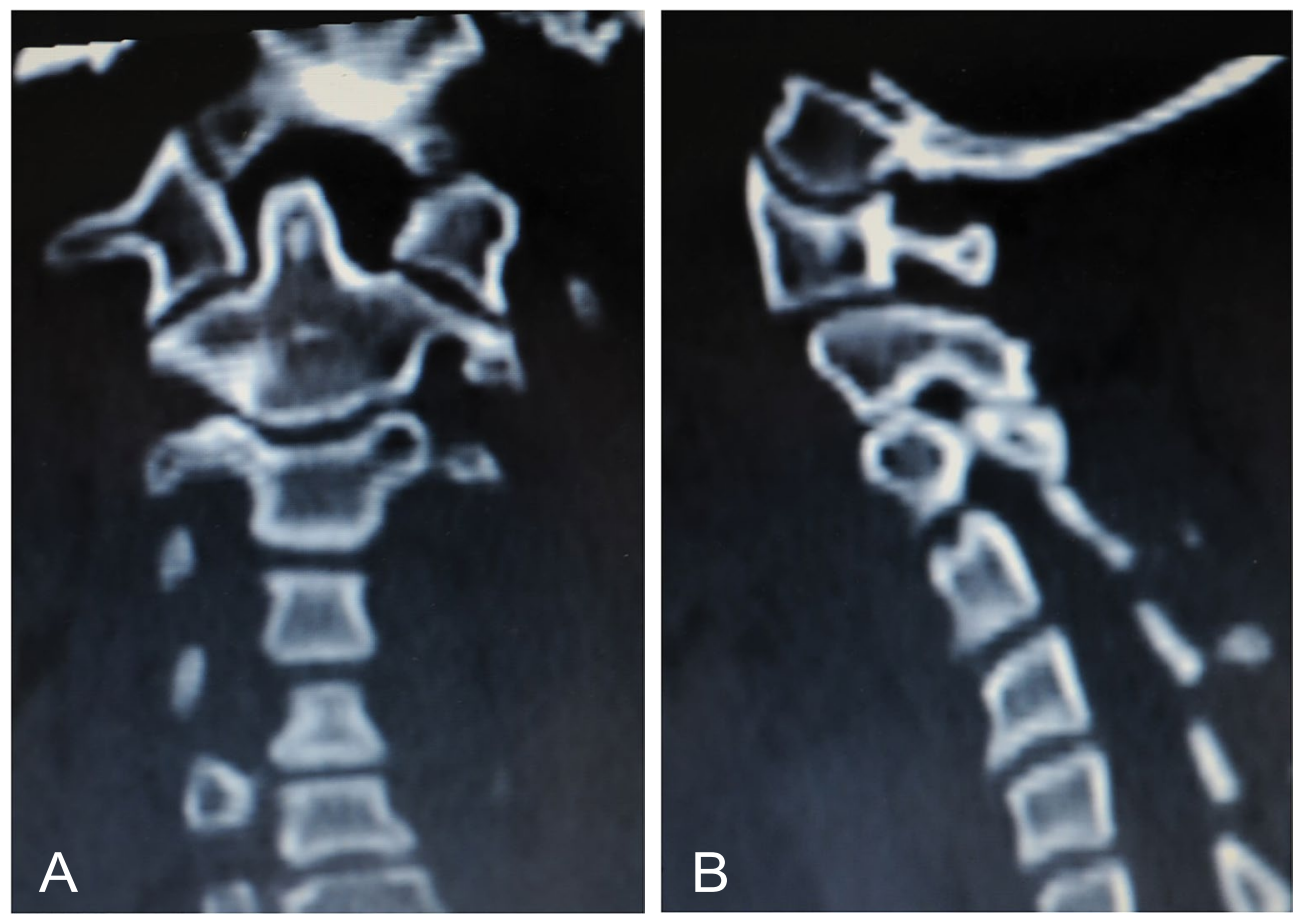

Figure 3: 2D CT: a) A coronal plane search found odontoid apophysis symmetry regarding lateral masses of the atlas; b) A sagittal plane search found an occipital condyle of normal morphology.
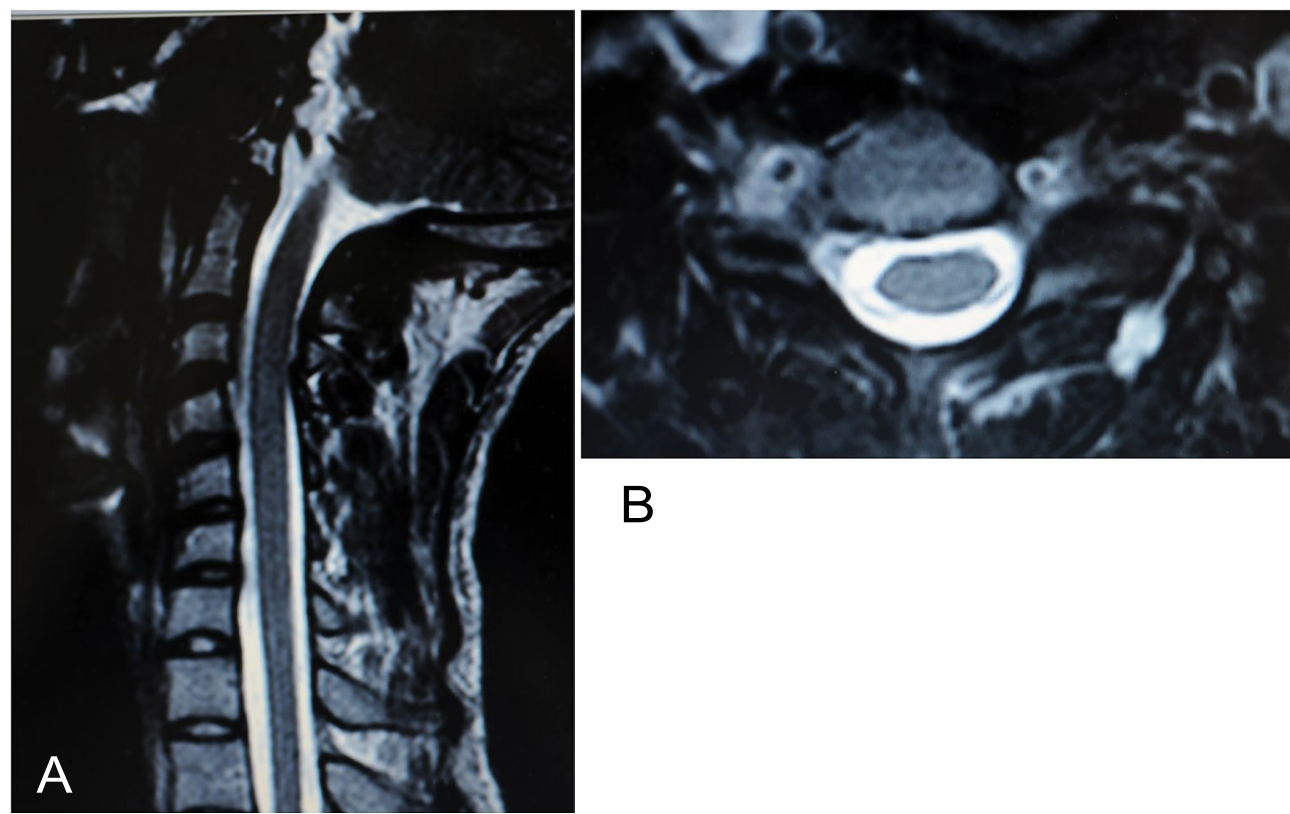

B

Figure 4: MR-scanning: a) Sagittal plane: A normal spinal cord in the cervical spinal canal; b) Axial plane: Spinal canal asymmetry was marked by a displaced spinal cord to the left (i.e., toward the concavity of the torticollis).

icant changes in preoperative vertebral bone deformities, either visually or through the morphology of the spinal canal (Figure 5a, Figure 5b, and Figure 6).

\section{Discussion}

Torticollis requires the differential diagnosis of congenital pathologies syndromes (such as Klippel-Feil, Down, Marfan, and Morquio syndromes and diastematomyelia), infectious diseases (Grisel's Syndrome and mastoiditis), inflammatory conditions (rheumatoid juvenile arthritis), gastroesophageal syndromes (Salinger Syndrome) and tumors (osteoblastoma, osteoid osteoma, posterior fos- sa astrocytoma, etc.) as well as excluding trauma to the occipito-alto-odontoid hinge $[1,2,4,5]$. In the adolescent case upon which we report, all were excluded based on confirming infantile inveterate muscular torticollis by clinical history. The spine development depends of somites. This embryonic structures develop segmentally vertebrae. Failure of this segmental horizontal develop may be mechanism that leads to congenital scoliosis [6]. At the age of 5-6 years, fusion of the anterior arches of the atlas and of the odontoid apophysis of the axis had occurred $[7,8]$. Atlas and axis has, both, two neurocentral synchondoses. Mechanical forces influencing 


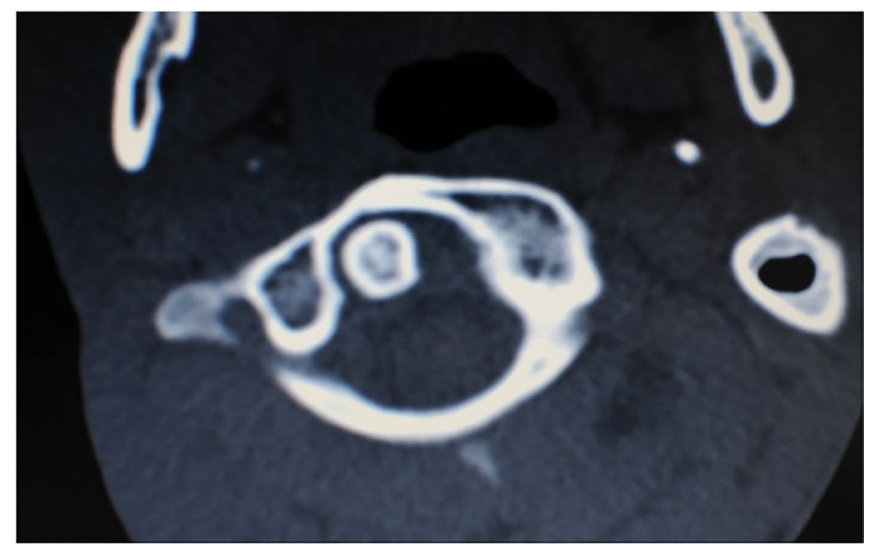

A
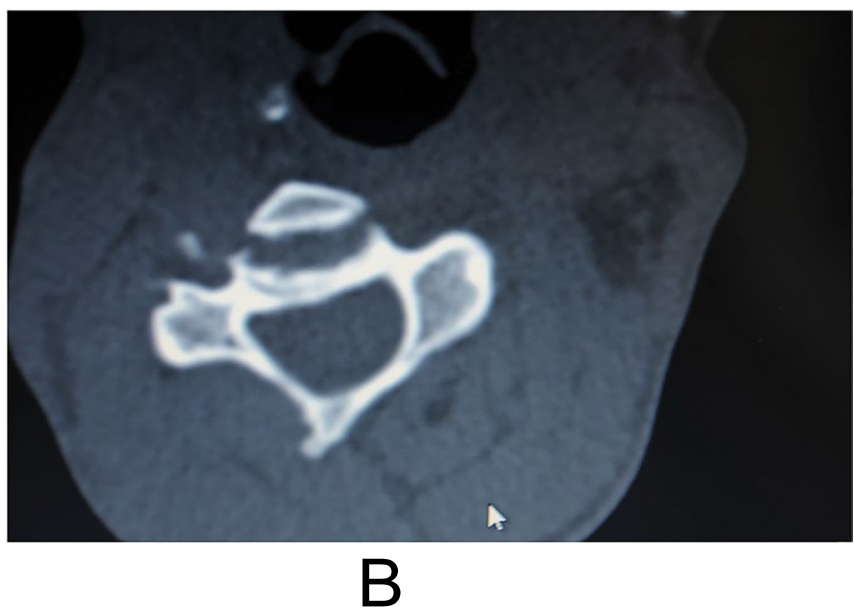

Figure 5: a) Postoperative CT (5 years follow-up); b) Persistent abnormal positions of odontoid apophysis and asymmetry of the posterior vertebral arches and cervical spinal canal.

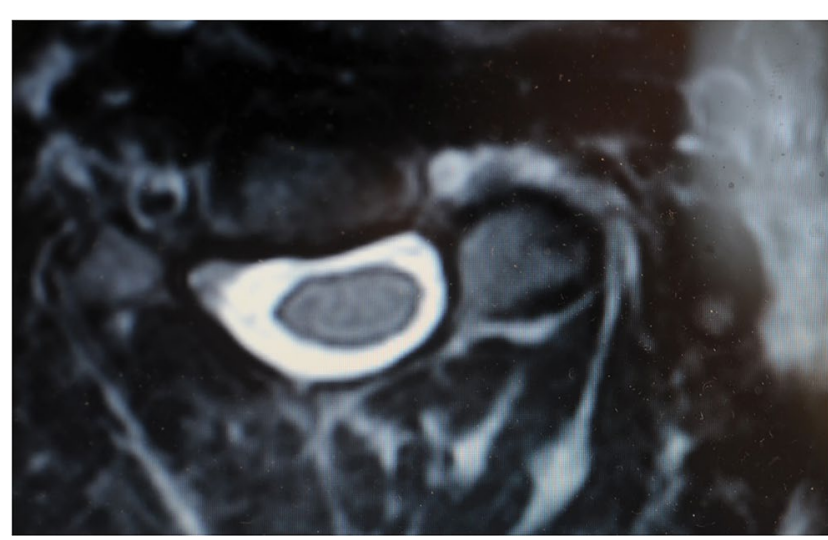

Figure 6: Postoperative MR-scanning (5 years) remained unchanged: Spinal canal deformity and the asymmetric position of the spinal cord towards the concavity of the torticollis are noted.

in this neurocentral synchondoses, can cause abnormal and asymmetric develop in neurocentral synchondoses or in periosteal sleeve of posterior vertebrae elements [6]. The patient was diagnosed and treated by us at age 9. The CT and MRI showed deformities that were associated with torticollis; these deformities have not been described previously in the literature but are similar to those that occur in scoliosis in vertebrae (pedicles and posterior arches) and in the asymmetrical position of the spinal cord in the spinal canal. These deformities in scoliosis were attributed to the sustained action of deforming forces, especially shear stress forces, on the neurocentral synchondroses in other regions of the column [9]. In recent years, have been published manuscripts about vertebral wedging deformity, especially, in frontal plane and pedicle and posterior elements changes [10-12]. Other authors, have published about characteristic and important 3D vertebrae deformity pattern in scoliosis, with the objective to know better the deformities and to be able to improve the surgery in scoliotic spine [13-17]. At 5 years, tenotomy of the SCM showed no indication of significant changes in preoperative deformities, including facial asymmetry [18]. We could propose two hypotheses: a) The persistent muscular deforming forces, from postnatal age, originated from bone and proximal cervical spinal canal deformities and b) Rotating deforming forces also acted on the cervical spine, similar to what occurs in scoliosis, a finding that extends to the rest of the spinal column $[18,19]$. For readers, limitation of hypotheses is evident, because is a case report, but persistent mechanical shear stress forces in a torticollis, could be a possible cause of damage on neurocentral synchondroses and develop 3D scoliosis vertebrae deformities.

\section{Conflict of Interest}

No conflict of interest.

\section{References}

1. Ballock RT, Song KM (1996) The prevalence of nonmuscular causes of torticollis in children. J Pediatr Orthop 16: 500-504.

2. Do TT (2006) Congenital muscular torticollis: Current concepts and review of treatment. Curr Opin Pediatr 18: 26-29.

3. Herman MJ (2006) Torticollis in infants and children: common and unusual causes. Instr Course Lect 55: 647-653.

4. Haque S, Shafi BB, Kaleem M (2012) Imaging of torticollis in children. Radiographics 32: 557-571.

5. Tachibana A, Imabayashi H, YatoY, Nakamichi K, Asazuma T, et al. (2010) Torticollis of a specific C1 dislocation with split atlas. Spine (Phila Pa 1976) 35: E672-E675.

6. Weinstein SL (2001) The pediatric spine. Principles and Practice. ( $2^{\text {nd }}$ edn), Lippincott Williams \& Wilkins, Philadelphia.

7. Al Kaissi A, Ganger R, Hofstaetter JG, KlaushoferK, Grill F (2011) The aetiology behind torticollis and variable spine defects in patients with Müllerian duct/renal aplasia- cervicothoracic somite displasia síndrome: 3D CT scan analysis. Eur Spine J 20: 1720-1727.

8. Sarwahi V, Sugarman EP, Wollowick AL, Amaral TD, Lo $Y$, et al. (2014) Prevalence, distribution, and surgical relevance of abnormal pedicles in spines with adolescent idiophatic scoliosis vs. No deformity. A CT- based study. J Bone Joint Surg Am 96: e92.

9. Samdani AF, Williams RC, Danish S, Betz R (2009) Torticollis manifest after a minor fall with underlying bony anomalies and a hypoplastic vertebral artery. J Pediatr Orthop B 18: $271-274$ 
10. Parent S, Labelle H, Skalli W, de Guise J (2004) Vertebral wedging characteristic changes in scoliotic spines. Spine (Phila Pa 1976) 29: e455-e462.

11. Parent S, Labelle $H$, Mitulescu A, Latimer B, Skalli W, et al. (2002) Morphometric analysis of one anatomic scoliotic specimen. Stud Health Technol Inform 88: 387-392.

12. Parent S, Labelle H, Skalli W, Latimer B, de Guise J (2002) Morphometric analysis of anatomic scoliotic specimens. Spine (Phila Pa 1976) 27: 2305-2311.

13. Shen J, Kadoury S, Labelle H, Parent S (2016) Geometric Torsion in Adolescent Idiopathic Scoliosis: A Surgical Outcomes Study of Lenke Type 1 Patients. Spine (Phila Pa 1976) 41: 1903-1907.

14. Shen J, Kadoury S, Labelle H, Roy-Beaudry M, Aubin CÉ, et al. (2017) Geometric Torsion in Adolescent Idiopathic Scoliosis: A New Method to Differentiate Between Lenke 1 Subtypes. Spine (Phila Pa 1976) 42: E532-E538.

15. Le Navéaux F, Aubin CE, Parent S, O Newton P, Labelle H (2017) 3D rod shape changes in adolescent idiopathic sco- liosis instrumentation: How much does it impact correction? Eur Spine J 26: 1676-1683.

16. Benameur S, Mignotte M, Parent S, Labelle H, Skalli W, et al. (2002) 3D biplanar statistical reconstruction of scoliotic vertebrae. Stud Health Technol Inform 91: 281-285.

17. Henao J, Aubin CÉ, Labelle H, Arnoux PJ (2016) Patient-specific finite element model of the spine and spinal cord to assess the neurological impact of scoliosis correction: preliminary application on two cases with and without intraoperative neurological complications. Comput Methods Biomech Biomed Engin 19: 901-910.

18. Lee JK, Moon HK, Park MS, Yoo WJ, Choi IH, et al. (2012) Change of craniofacial deformity after sternocleidomastoid muscle release in pediatric patients with congenital muscular torticollis. J Bone Joint Surg Am 94: e93.

19. Çatan H, Buluc L, Anık Y, Ayyıldız E, Sarlak A (2007) Pedicle morphology of the thoracic spine in preadolescent idiopathic scoliosis: Magnetic resonance supported analysis. Eur Spine J 16: 1203-1208. 